\title{
GW23-e1250 WHAT IMPACTS DOOR-TO-BALLOON TIME IN REMOTE AREA OF CHINA-AN ANALYSIS FROM A SINGLE CENTRE
}

doi:10.1136/heartjnl-2012-302920l.28

Xin Shen, Zixiang Yu, Yitong Ma. 1st Affiliated Hospital of Xinjiang Medical University

Objectives Our study was aim to analyse the impact factors and identified which factors significantly prolonged the DTB time in the west of China.

Methods We analysed the DTB time and its components from January 2008 to December 2010 in 301 consecutive patients presenting with STEMI in our hospital. Then, we determined which factors significantly prolonged the DTB time.

Results The median DTB time of all the patients was $149 \pm 78 \mathrm{~min}$, the group was divided by DTB time, the $\leq 120 \mathrm{~min}$ group and the $>120$ min group. The median DTB time of two groups were 87 $\pm 29 \mathrm{~min}$ and $201 \pm 68 \mathrm{~min}$ respectively $(p=0.000)$. In the $\leq 120 \mathrm{~min}$ group, more patients $(68.1 \%)$ presented to our hospital during working hours $(p=0.000)$ while in the $>120$ min group more patients $(63.2 \%)$ presented during off hours $(p=0.000)$. Moreover, more patients $(49.3 \%)$ presented when the interventional doctors was on site $(p=0.000)$ in the $\leq 120$ min group. Factors that significantly lengthened DTB time included the prolonged time of consultation by cardiology doctor $(p=0.000)$, the prolonged time of explaining patient's condition to the family $(p=0.000)$, interventional doctor was off duty $(p=0.000)$, having the symptom of

slight chest pain $(p=0.000)$, presenting during off hours $(\mathrm{p}=0.000)$.

Conclusions The tertiary care general hospital in the remote area, the prolonged time of consultation by cardiology doctor and explaining patient's condition to the family really accounted for the majority of reperfusion delay. Besides, lacking of interventional doctors usually resulted in DTB time delay during off hours. Therefore, directly activating the catheterisation laboratory by emergency department, strengthening the doctor to know the seriousness of early reperfusion, spreading propaganda of PCI among the masses, and increasing the numbers of interventional doctors will shorten DTB time more. 